\title{
Independent nonclassical tests for states and measurements in the same experiment
}

\author{
Alfredo Luis ${ }^{1}$ and Ángel Rivas ${ }^{2}$ \\ ${ }^{1}$ Departamento de Óptica, Facultad de Ciencias Físicas, Universidad Complutense, \\ 28040 Madrid, Spain \\ ${ }^{2}$ Institut für Theoretische Physik, Universitt Ulm, Ulm D-89069, Germany \\ E-mail: alluis@fis.ucm.es
}

\begin{abstract}
We show that one single experiment can test simultaneously and independently both the nonclassicality of states and measurements by the violation or fulfillment of classical bounds on the statistics. Nonideal measurements affected by imperfections can be characterized by two bounds depending on whether we test the ideal measurement or the real one.
\end{abstract}

Keywords: Nonclassical states, quantum optics 


\section{Introduction}

Within standard quantum theory, quantum states play two dissimilar but complementary roles: (i) they express the state of the system, represented by a density matrix $\rho$, and (ii) they determine the statistics of measurements, typically by projection of the system state on the eigenstates of the measured observable. More precisely, any observable event is represented by a nonnegative Hermitian operator $\Delta$ (maybe part of a larger positive operator-valued measure) that determines the event probability as $p=\operatorname{tr}(\rho \Delta)$, where $\rho$ is the state of the system. In many relevant situations $\Delta$ is proportional to a suitable state, such as photon-number and quadrature measurements in quantum optics. Positive operators playing the role of $\Delta$ can be turned into the role $\rho$ as shown in [1].

While referring to nonclassical states is quite common, not so many effort has been devoted to nonclassical measurements [2, 3, 4, 5]. A customary criterion of nonclassicality for states $\rho$ is the failure of the Glauber-Sudarshan $P$ function to exhibit all the properties of a classical probability density [6]. This occurs when $P$ takes negative values, or when it fails to be a proper function becoming more singular than the delta function. Accordingly, we may say that the event represented by $\Delta$ in Hilbert space is nonclassical when its $P$ phase-space representative takes negative values or is more singular than the delta function.

Although the nonclassicality of states and measurements are different things, both may be tested simultaneously within one single experiment in terms of its statistics $p$. This possibility is addressed in this work by means of a simple example: nonefficient single-photon detection in photon-added thermal states [7]. A key point of this example is feasibility, since these states have been already generated in experiment [8], and it explicitly includes typical imperfections such as losses and thermalization.

As nonclassicality criteria we will consider the simple and robust practical tests recently introduced where nonclassicality is revealed by breaking classical bounds on probabilities satisfied by all classical states and measurements [4, 9] (see [10] for other nonclassicality criteria). The main features of these tests are recalled in section 2 .

\section{Classical bounds on probabilities}

For definiteness let us focus on a single mode of the electromagnetic field with complexamplitude operator $a$. To derive the nonclassical tests we will use the $P$ and $Q$ phasespace representatives associated to any operator $A$

$$
A=\int \mathrm{d}^{2} \alpha P_{A}(\alpha)|\alpha\rangle\langle\alpha|, \quad Q_{A}(\alpha)=\frac{1}{\pi}\langle\alpha|A| \alpha\rangle,
$$

where $|\alpha\rangle$ are coherent states, $a|\alpha\rangle=\alpha|\alpha\rangle$. They are suitably normalized

$$
\int \mathrm{d}^{2} \alpha P_{A}(\alpha)=\int \mathrm{d}^{2} \alpha Q_{A}(\alpha)=\operatorname{tr} A
$$

with $\mathrm{d}^{2} \alpha=\mathrm{d} x \mathrm{~d} y$, where $x, y$ are the real and imaginary parts of $\alpha=x+\mathrm{i} y$. 
Exploiting the $\rho \leftrightarrow \Delta$ symmetry, the same probability $p=\operatorname{tr}(\rho \Delta)$ can be expressed by two equivalent formulas

$$
p=\pi \int \mathrm{d}^{2} \alpha P_{\rho}(\alpha) Q_{\Delta}(\alpha)=\pi \int \mathrm{d}^{2} \alpha P_{\Delta}(\alpha) Q_{\rho}(\alpha) .
$$

By using the first equality we are able to derive bounds sensitive to the nonclassicality of the state $\rho$, while the second equality leads to bounds sensitive to the nonclassicality of the measurement $\Delta$. Note that the $Q$ function is always a positive and well behaved.

\subsection{Nonclassical test for states}

For classical states, i. e., for ordinary nonnegative functions $P_{\rho}(\alpha) \geq 0$, we get

$$
P_{\rho}(\alpha) Q_{\Delta}(\alpha) \leq P_{\rho}(\alpha) Q_{\Delta, \max }
$$

where $Q_{\Delta, \max }$ is the maximum of $Q_{\Delta}(\alpha)$ when $\alpha$ is varied. Applying this to the first equality in (3), and taking into account (2), we get the following upper bound $S$ for $p$

$$
p \leq S=\pi Q_{\Delta, \max },
$$

that holds for every $P_{\rho}(\alpha)$ compatible with classical physics. If this condition is violated it means that (4) is false and the state is not classical.

\subsection{Nonclassical test for measurements}

For classical measurements, i. e., for ordinary nonnegative function $P_{\Delta}(\alpha) \geq 0$ it holds that

$$
P_{\Delta}(\alpha) Q_{\rho}(\alpha) \leq P_{\Delta}(\alpha) Q_{\rho, \max },
$$

where $Q_{\rho, \max }$ is the maximum of $Q_{\rho}(\alpha)$ when $\alpha$ is varied. Applying this to the second equality in (3) we get the following upper bound $M$ for $p$, provided that $\operatorname{tr} \Delta$ is finite,

$$
p \leq M=\pi Q_{\rho, \max } \operatorname{tr} \Delta .
$$

Equation (7) can be violated if $P_{\Delta}(\alpha)$ fails to be positive or when it becomes a generalized function (this is a nonclassical measurement) since in both cases (6) fails to be true.

\section{Inefficient photon detection on photon-added thermal states}

The same probability $p$ may serve to test both the nonclassicality of $\rho$ and $\Delta$. Let us demonstrate this by applying the above formalism to nonefficient single-photon detection in photon-added thermal states. 


\subsection{Single-photon-added thermal states}

The single-photon-added thermal states read, in the photon-number basis, [7, 8]

$$
\rho=(1-\xi) a^{\dagger} \rho_{\mathrm{tc}} a=(1-\xi)^{2} \sum_{n=1}^{\infty} \xi^{n-1} n|n\rangle\langle n|,
$$

where $\rho_{\text {tc }}$ is a thermal chaotic state

$$
\rho_{\mathrm{tc}}=(1-\xi) \sum_{n=0}^{\infty} \xi^{n}|n\rangle\langle n|,
$$

with mean number of photons

$$
\bar{n}=\frac{\xi}{1-\xi} .
$$

The $P$ representative of $\rho$ is well-behaved but nonpositive

$$
P_{\rho}(\alpha)=\frac{1}{\pi \bar{n}^{3}}\left[(\bar{n}+1)|\alpha|^{2}-\bar{n}\right] \exp \left(-\frac{|\alpha|^{2}}{\bar{n}}\right),
$$

while the $Q$ function is

$$
Q_{\rho}(\alpha)=\frac{|\alpha|^{2}}{\pi(\bar{n}+1)^{2}} \exp \left(-\frac{|\alpha|^{2}}{\bar{n}+1}\right)
$$

so that its maximum occurs for $|\alpha|^{2}=\bar{n}+1$ being

$$
Q_{\rho, \max }=\frac{1}{\pi \mathrm{e}(\bar{n}+1)}
$$

These states present three relevant features for our purposes:

(i) Their nonclassical behavior is independent of other typical nonclassical features, since there is no quadrature squeezing, they present super-Poissonian photon-number statistics for all $\bar{n}>1 / \sqrt{2}$, and have no oscillatory statistics [4].

(ii) They can be generated experimentally [8].

(iii) Their definition embodies a typical source of practical imperfection such as thermalization.

\subsection{Ideal single-photon detection}

For ideal single-photon detection we have, in the photon-number basis, $\Delta=|1\rangle\langle 1|$ with $\operatorname{tr} \Delta=1$. The $P$ representative is nonclassical being more singular than the delta function

$$
P_{\Delta}(\alpha)=\left(1+\frac{\partial^{2}}{\partial \alpha \partial \alpha^{*}}\right) \delta^{(2)}(\alpha) .
$$

The $Q$ function is

$$
Q_{\Delta}(\alpha)=\frac{|\alpha|^{2}}{\pi} \exp \left(-|\alpha|^{2}\right)
$$

and the maximum occurs at $|\alpha|=1$

$$
Q_{\Delta, \max }=\frac{1}{\mathrm{e} \pi} \text {. }
$$




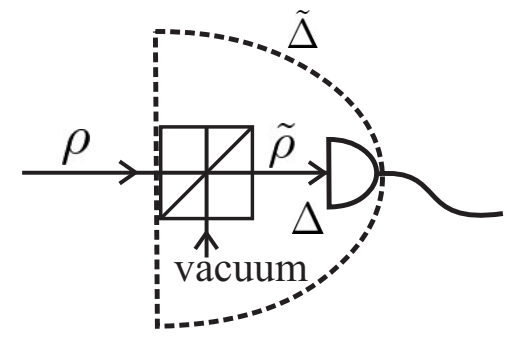

Figure 1. Illustration of inefficient single-photon detection.

If the measured state is classical, the single-photon probability $p$ is bounded by [4, 9]

$$
p \leq S=\frac{1}{\mathrm{e}} .
$$

\subsection{Inefficient single-photon detection}

In figure 1 we illustrate the case of inefficient single-photon detection. A detector with quantum efficiency $\eta$ can be modeled by a beam splitter of amplitude-transmission coefficient $t=\sqrt{\eta}$, mixing the input state $\rho$ with vacuum, placed before an ideal detector $\Delta$ with $\eta=1$ [11]. After this model two different routes can be followed:

(i) We can test the underlying ideal detection $\Delta$ regarding inefficiency as a handicap of practical origin. This is to say that we have ideal detection on the state $\tilde{\rho}$ after the beam splitter that carries the effect of inefficiency, so that the probability is $p=\operatorname{tr}(\tilde{\rho} \Delta)$. Since the transformation of coherent states through lossless beam splitters is $|\alpha\rangle \rightarrow|\sqrt{\eta} \alpha\rangle$ the state $\tilde{\rho}$ is

$$
\tilde{\rho}=\int \mathrm{d}^{2} \alpha P_{\rho}(\alpha)|\sqrt{\eta} \alpha\rangle\langle\sqrt{\eta} \alpha|,
$$

where $P_{\rho}(\alpha)$ is in (11). From this expression we get by direct computation the $Q$ function of $\tilde{\rho}$

$$
Q_{\tilde{\rho}}(\alpha)=\frac{1}{\pi}\left[\frac{(\bar{n}+1) \eta|\alpha|^{2}}{(\eta \bar{n}+1)^{3}}+\frac{1-\eta}{(\eta \bar{n}+1)^{2}}\right] \exp \left(-\frac{|\alpha|^{2}}{\eta \bar{n}+1}\right) .
$$

For $\eta \neq 0$ its maximum holds for

$$
|\alpha|^{2}=1+\eta \bar{n}-\frac{(1-\eta)(1+\eta \bar{n})}{\eta(\bar{n}+1)},
$$

leading to

$$
Q_{\tilde{\rho}, \max }=\frac{\eta(\bar{n}+1)}{\pi(\eta \bar{n}+1)^{2}} \exp \left[-\frac{\eta \bar{n}+2 \eta-1}{\eta(\bar{n}+1)}\right] .
$$

(ii) Alternatively, we can test the real measurement embodying the inefficiency as part of the measuring apparatus. The real measurement is represented by an Hermitian nonnegative operator $\tilde{\Delta}$ to be determined such that the probability can be expressed as $p=\operatorname{tr}(\rho \tilde{\Delta})$. From (3), (18) and the equality $p=\operatorname{tr}(\tilde{\rho} \Delta)=\operatorname{tr}(\rho \tilde{\Delta})$ we get

$$
Q_{\tilde{\Delta}}(\alpha)=Q_{\Delta}(\sqrt{\eta} \alpha)=\frac{\eta|\alpha|^{2}}{\pi} \exp \left(-\eta|\alpha|^{2}\right),
$$


so that

$$
Q_{\tilde{\Delta}, \max }=Q_{\Delta, \max }=\frac{1}{\pi \mathrm{e}} .
$$

From (2), (22) and $\operatorname{tr} \Delta=1$ we readily get

$$
\operatorname{tr} \tilde{\Delta}=\frac{1}{\eta} \text {. }
$$

Moreover, by expressing the exponential in (22) as $\exp \left(-\eta|\alpha|^{2}\right)=\exp [(1-$ $\left.\eta)|\alpha|^{2}\right] \exp \left(-|\alpha|^{2}\right)$ and expanding the first exponential in power series we get

$$
Q_{\tilde{\Delta}}(\alpha)=\frac{\eta}{\pi} \sum_{n=0}^{\infty}(n+1)(1-\eta)^{n} \frac{|\alpha|^{2(n+1)}}{(n+1) !} \exp \left(-|\alpha|^{2}\right),
$$

that readily provides the expression of $\tilde{\Delta}$ in the photon-number basis

$$
\tilde{\Delta}=\eta \sum_{n=0}^{\infty}(n+1)(1-\eta)^{n}|n+1\rangle\langle n+1|=\eta(1-\eta)^{a^{\dagger} a-1} a^{\dagger} a .
$$

The probability $p$ is independent of the interpretations (i) and (ii), being [from (8) and (26) for example]

$$
p=\operatorname{tr}(\tilde{\rho} \Delta)=\operatorname{tr}(\rho \tilde{\Delta})=\eta \frac{1+2 \bar{n}-\eta \bar{n}}{(1+\eta \bar{n})^{3}} .
$$

The interpretations also do not affect the classical upper bound for states, from (5) and (23),

$$
p \leq S=\pi Q_{\Delta, \max }=\frac{1}{\mathrm{e}} .
$$

The interpretations (i)/(ii) affect the classical upper bound for measurements. Since we are actually considering two different measurements, the ideal $\Delta$ and the real $\tilde{\Delta}$, we get from (13), (21), and (24) two different classical bounds $M_{\Delta}$ and $M_{\tilde{\Delta}}$ with

$$
p \leq M_{\Delta}=\pi Q_{\tilde{\rho}, \max } \operatorname{tr} \Delta=\frac{\eta(\bar{n}+1)}{(\eta \bar{n}+1)^{2}} \exp \left[-\frac{\eta \bar{n}+2 \eta-1}{\eta(\bar{n}+1)}\right]
$$

and

$$
p \leq M_{\tilde{\Delta}}=\pi Q_{\rho, \max } \operatorname{tr} \tilde{\Delta}=\frac{1}{\mathrm{e} \eta(\bar{n}+1)} .
$$

In figure 2 we have plotted $p, M_{\tilde{\Delta}}, M_{\Delta}$, and $S$ as functions of $\bar{n}$ for $\eta=0.4$ and 0.9 , while in figure 3 they are plotted as functions of $\eta$ for $\bar{n}=0.2$ and 0.7 . Several conclusions can be derived from these plots:

(a) $M_{\tilde{\Delta}}$ is always above $M_{\Delta}$, so that it is more difficult to prove the nonclassicality of the real $\tilde{\Delta}$ than of the ideal $\Delta$. This fits with the general idea that inefficiencies degrade quantum properties.

(b) When $\eta$ increases $M_{\tilde{\Delta}}$ and $M_{\Delta}$ become closer. This may be expected since when $\eta \rightarrow 1$ we get $\tilde{\Delta} \rightarrow \Delta$.

(c) In general $p$ decreases when $\bar{n}$ increases, enforcing the fulfillment of the classical bounds. This agrees with common understanding of the effect of thermalization. An 

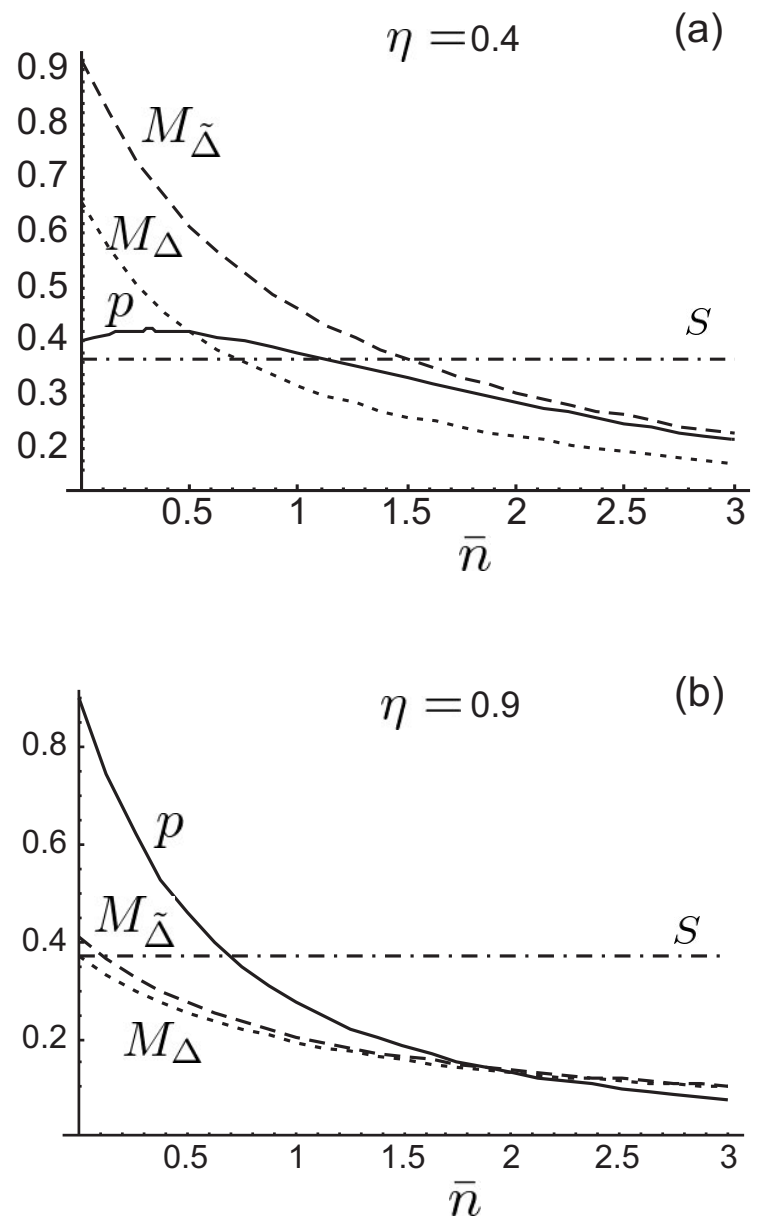

Figure 2. Plots of $p$ (solid), $M_{\tilde{\Delta}}$ (dashed), $M_{\Delta}$ (dotted), and $S$ (dash-dotted), as functions of $\bar{n}$ for fixed $\eta=0.4$ (a) and $\eta=0.9$ (b).

exception occurs when $\eta$ is rather low, since for small $\eta$ thermal photons may increase the probability of photon detection, as illustrated in figure 2(a).

(d) For low values of $\bar{n}$ increasing $\eta$ favors the violation of the classical bounds by increasing $p$ and decreasing $M_{\tilde{\Delta}}$ and $M_{\Delta}$, as illustrated in figure 3(a). On the other hand, for larger $\bar{n}$ we get that larger $\eta$ increases the probability of detecting more than a single photon, decreasing $p$ as illustrated in figure $3(\mathrm{~b})$.

(e) For large $\eta$ and small $\bar{n}$ it is possible to have $p>M_{\tilde{\Delta}}, M_{\Delta}, S$ simultaneously, so that one and the same measurement can reveal at the same time the nonclassical character of the $\rho, \Delta$, and $\tilde{\Delta}$ as illustrated in figures $2(\mathrm{~b})$ and $3(\mathrm{a})$.

\section{Conclusions}

We have shown that the same experiment can test simultaneously and independently both the nonclassicality of states and measurements. This is because the nonclassicality of states and measurements manifests via the violation of different and independent bounds to the same statistics. We have shown that practical imperfections produce the 

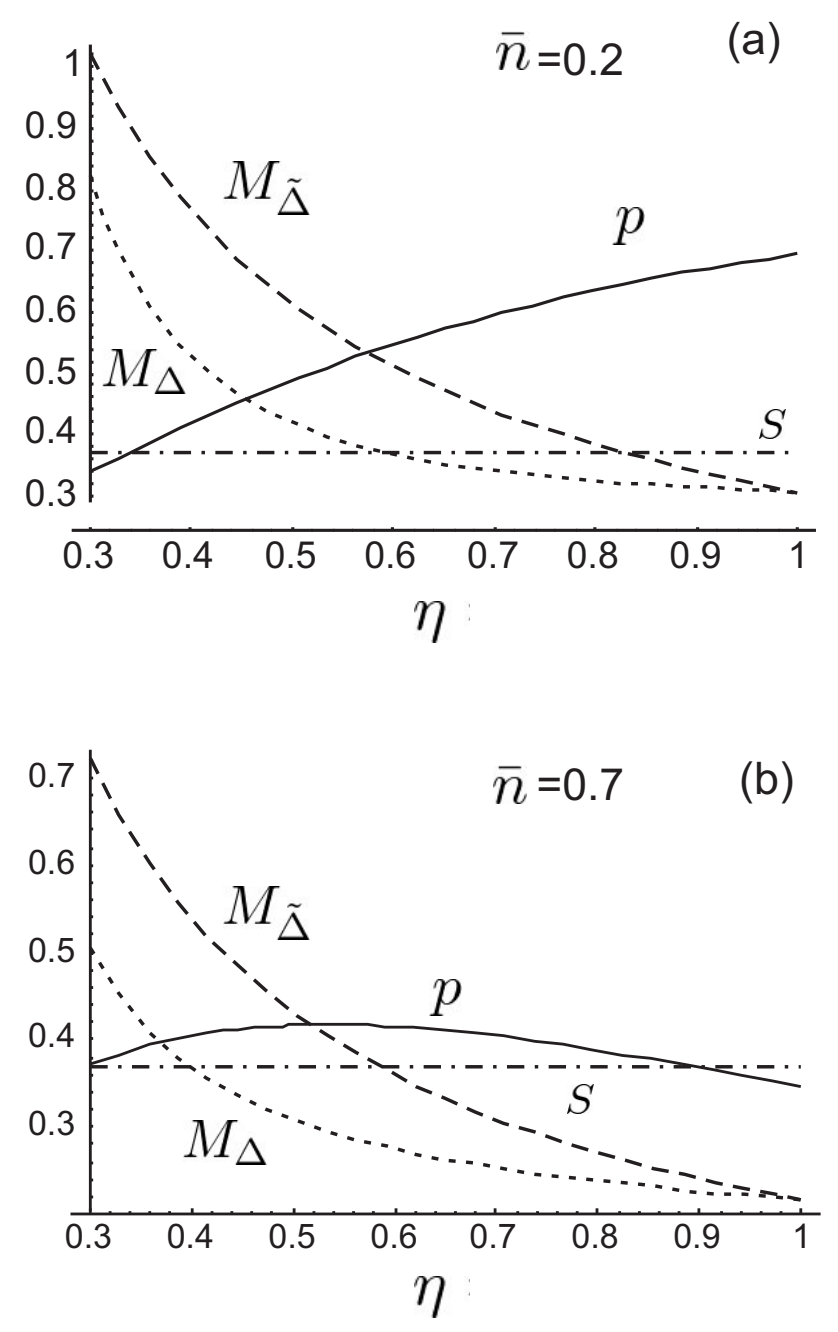

Figure 3. Plots of $p$ (solid), $M_{\tilde{\Delta}}$ (dashed), $M_{\Delta}$ (dotted), and $S$ (dash-dotted), as functions of $\eta$ for fixed $\bar{n}=0.2$ (a) and $\bar{n}=0.7$ (b).

existence of two bounds testing the ideal and real measurements.

\section{Acknowledgments}

A. R. acknowledges financial support from the EU Integrated Project QESSENCE and the STREP action CORNER. A. L. acknowledges support from project No. FIS2008-01267 of the Spanish Dirección General de Investigación del Ministerio de Ciencia e Innovación, and by project QUITEMAD S2009-ESP-1594 of the Consejería de Educación de la Comunidad de Madrid.

\section{References}

[1] Luis A and Sánchez-Soto L L 1998 Phys. Lett. A 244211

[2] Luis A and Sánchez-Soto L L 1999 Phys. Rev. Lett. 833573 
Fiurášek J 2001 Phys. Rev. A 64024102

[3] Coldenstrodt-Ronge H B, Lundeen J S, Pregnell K L, Feito A, Smith B J , Mauerer W, Silberhorn Ch, Eisert J, Plenio M B, and Walmsley I A 2009 J. Mod. Opt. 56432

Lundeen J S, Feito A, Coldenstrodt-Ronge H, Pregnell K L, Silberhorn Ch, Ralph T C, Eisert J, Plenio M B, and Walmsley I A 2009 Nature Physics 527

[4] Rivas A and Luis A 2009 Phys. Rev. A 79042105

[5] Amri T, Laurat J and Fabre C arXiv:1006.5882.

Amri T, e-print arXiv:1001.3032

[6] Mandel L and Wolf E 1995 Optical Coherence and Quantum Optics (Cambridge, England: Cambridge University Press)

Scully M O and Zubairy M S 1997 Quantum Optics (Cambridge, England: Cambridge University Press)

Gerry C C and Knight P L 2005 Introductory Quantum Optics (Cambridge, England: Cambridge University Press)

Dodonov V V 2002 J. Opt. B: Quantum Semiclass. Opt. 4 R1

[7] Agarwal G S and Tara K 1992 Phys. Rev. A 46485

[8] Zavatta A, Parigi V and Bellini M 2007 Phys. Rev. A 75052106

Kiesel T, Vogel W, Parigi V, Zavatta A and Bellini M 2008 Phys. Rev. A 78 021804R

[9] Hillery M 1987 Phys. Rev. A 35725

[10] Mandel L 1986 Phys. Scr. T12 34

Lee C T 1991 Phys. Rev. A 44 R2775

Benedict M G and Czirják A 1999 Phys. Rev. A 604034

Dodonov V V, Man'ko O V, Man'ko V I, and Wünsche A 2000 J. Mod. Opt. 47633

Vogel W 2000 Phys. Rev. Lett. 841849

Hall M J W 2000 Phys. Rev. A 62012107

Marian P, Marian T A, and Scutaru H 2002 Phys. Rev. Lett. 88153601

Malbouisson J M C and Baseia B 2003 Phys. Script. 6793

Dodonov V V and Renó M B 2003 Phys. Lett. A 308249

Marian P, Marian T A, and Scutaru H 2004 Phys. Rev. A 69022104

Asbóth J K, Calsamiglia J, and Ritsch H 2005 Phys. Rev. Lett. 94173602

Giraud O, Braun P and Braun D 2008 Phys. Rev. A 78042112

Miranowicz A, Bartkowiak M, Wang X, Liu Y, and Nori F 2010 Phys. Rev. A 82013824

Sperling J and Vogel W e-print arXiv:1004.1944v1

[11] Yuen H P and Shapiro J H 1980 IEEE Trans. Inf. Theory IT-26 78

Yurke B 1985 Phys. Rev. A 32311

Leonhardt U and Paul H 1993 Phys. Rev. A 484598

Kim T, Ha Y, Shin J, Kim H, Park G, Kim K, Noh T G, and Hong Ch K 1999 Phys. Rev. A 60708

Semenov A A, Turchin A V, and Gomonay H V 2008 Phys. Rev. A 78055803 